This is the author's version of an article published in

Statistics, Vol. 46, No. 4, August 2012, 549-561

It is available online at doi:10.1080/02331888.2010.543463

\title{
Biased and Unbiased Estimation of the Circular Mean Resultant Length and its Variance
}

\author{
Rade Kutil \\ University of Salzburg, Department of Computer Sciences, \\ Jakob Haringer Str. 2, 5020 Salzburg, Austria \\ (Received 28 October 2008; final version received 17 November 2010)

\begin{abstract}
The mean resultant length (MRL) is the length of the average of random vectors on the unit circle. It is used to measure the concentration of unimodal circular distributions. The sample MRL, as an estimator for the population MRL, has not been investigated thoroughly yet. This work examines the bias, variance and mean squared error of the MRL. Unbiased or near unbiased estimators are developed wherever possible for the squared and non-squared MRL as well as their variances and mean squared error. All estimators are tested numerically on four representative circular distributions.
\end{abstract}

Keywords: circular statistics; unbiased estimator; resultant length

\section{Introduction}

In circular statistics (see [1,2]), a random variable $X$ is defined on an interval which is thought of as cyclic. Such an interval can be scaled to the range $[0,2 \pi]$ and represented properly by the unit circle in the complex plane through the expression $e^{i X}$. To provide parameters equivalent to the expected value $E(X)$ and the variance $V(X)$, as defined in the linear case, for circular random variables in a similar fashion, one considers the complex expected value $E\left(e^{i X}\right)=\rho e^{i \mu}$. In this representation, $\mu$ is the mean direction, i.e. the circular average of $X$, and $\rho$ is a measure of concentration. It is called mean resultant length (MRL).

$$
\rho(X):=\left|E\left(e^{i X}\right)\right|
$$

If $X$ is maximally concentrated at a single value, then $\rho=1$. If there is no concentration at all, e.g. for the uniform distribution, then $\rho=0$. In this case the mean direction is undefined. Of course, $\rho$ can also be 0 for multimodal distributions, e.g. when $P(X=0)=P(X=\pi)=\frac{1}{2}$. Often the so-called circular variance $C V(X)=1-\rho(X)$ is used instead.

This work deals with the estimation of the MRL and the squared MRL $\rho^{2}(X)$. Curiously, the estimator of the MRL on a random sample, which is also called MRL, is more commonly known than the above definition. It is often denoted $\bar{R}$. However, we concentrate on its properties as an estimator, rather than just the descriptive measure. Therefore, we write it as $\hat{\rho}$ and define it as follows. Given a random sample $\mathbf{x}=\left\{x_{1}, x_{2}, \ldots, x_{n}\right\}$, the MRL 
estimator is

$$
\hat{\rho}(\mathbf{x}):=\frac{1}{n}\left|e^{i x_{1}}+e^{i x_{2}}+\ldots+e^{i x_{n}}\right|
$$

$\hat{\rho}$ is used in many fields. In meteorology and geology it determines tendencies in the direction of air (see [3]), water and land mass motion. In physiological psychology it is called phase locking index (PLI) (see [4]) and measures the coherency of phases in EEG signals at certain frequencies. Many other applications could be mentioned.

In many applications, the MRL is used to test if there is a strong preferred direction in measured data. It is argued that this is the case if the sample MRL exceeds a certain threshold which is chosen arbitrarily. However, due to the MRL's variance and bias, such a strategy can be misleading, especially for small samples. If the distribution is known, a proper statistical test can be applied. If not, then one has to use descriptive measures such as the MRL. In this case, the reliability of the MRL is crucial. Therefore, this work aims at shedding light on this problem and, if possible, improving the situation.

Because no such thing as a distribution-free confidence interval for the population MRL has yet been found, we must rely on other means to see what a given sample MRL implies for the population MRL. A big sample MRL might result from a big population MRL, but it might also result from bias and random variation of the sample MRL, both of which are stronger for small samples. Therefore, we will examine the bias, and estimate the variance of the sample MRL. The results will be evaluated for some representative distributions.

The properties of the squared MRL $\hat{\rho}^{2}$ in terms of moments and distribution have been studied by $[1,5,6]$ primarily for the purpose of statistical testing. From the moments the variance of $\hat{\rho}^{2}$ can be derived quite easily. However, if the distribution is unknown, the properties of $\hat{\rho}^{2}$ have to be estimated entirely from a given sample. Also, the properties of the non-squared MRL $\hat{\rho}$ are more difficult to determine.

In linear statistics there are more or less obvious methods to treat estimates such as average and variance and to determine the accuracy of these estimates, e.g. $V(\bar{X})=\frac{1}{n} V(X)$. However, to do such things for the MRL, is more complicated. This work aims at developing some methods for this purpose. First we will have a look at the bias of $\hat{\rho}$ and $\hat{\rho}^{2}$. Then we will calculate the variance of $\hat{\rho}^{2}$ and develop an estimator. Also, an estimator for the variance of the non-squared estimator $\hat{\rho}$ will be found, as well as one for the mean squared error (MSE) of $\hat{\rho}^{2}$. Attempts will be made to find unbiased estimates as far as possible. Unbiased estimates can be found for $\rho^{2}, V\left(\hat{\rho}^{2}\right)$ and $\operatorname{MSE}\left(\hat{\rho}^{2}\right)$, and near unbiased estimates can be found for $\rho$ and $V(\hat{\rho})$. Finally, it will be shown how to estimate $\rho^{2}$ and $V\left(\hat{\rho}^{2}\right)$ for a subset of a larger sample, which is important for comparing distinct samples. All estimators are tested numerically on four representative circular distributions, i.e. the uniform distribution, two von Mises distributions with a $\kappa$ of 1 and 3 , and a discrete distribution with $P(X=0)=\frac{3}{4}$ and $P\left(X=\frac{3 \pi}{4}\right)=\frac{1}{4}$.

\section{Bias}

Let us now look at the expected values of the MRL estimator $\hat{\rho}$. We will see that the MRL is a biased but consistent estimator, which gives us a first lower limit for the sample size necessary for proper estimates. Let $\mathbf{X}=\left\{X_{1}, X_{2}, \ldots, X_{n}\right\}$ be a set of independent random variables that own the same distribution as $X$. Then $E(\hat{\rho}(\mathbf{X}))$ is the expected estimate which would be equal to $\rho(X)$ if the MRL was unbiased. For ease of calculation, however, let us 
first examine $\hat{\rho}^{2}$ instead. We obtain

$$
\begin{aligned}
E\left(\hat{\rho}^{2}(\mathbf{X})\right) & =E\left(\frac{1}{n^{2}}\left|\sum_{k=1}^{n} e^{i X_{k}}\right|^{2}\right) \\
& =E\left(\frac{1}{n^{2}} \sum_{k=1}^{n} \sum_{l=1}^{n} e^{i X_{k}} e^{-i X_{l}}\right) \\
& =\frac{1}{n^{2}} E\left(\sum_{k=1}^{n} e^{i\left(X_{k}-X_{k}\right)}+\sum_{k=1}^{n} \sum_{l \neq k} e^{i X_{k}} e^{-i X_{l}}\right) \\
& =\frac{1}{n^{2}} \sum_{k=1}^{n} E(1)+\frac{1}{n^{2}} \sum_{k=1}^{n} \sum_{l \neq k} E\left(e^{i X_{k}} e^{-i X_{l}}\right) \\
& =\frac{n}{n^{2}}+\frac{n(n-1)}{n^{2}} \rho^{2}(X) \\
& =\frac{1}{n}+\frac{n-1}{n} \rho^{2}(X) .
\end{aligned}
$$

The derivation is presented here in such detail because the involved strategy, i.e. distinguishing between the cases $l=k$ and $l \neq k$, is applied later on in more involved circumstances. (3) shows that the squared MRL is biased, i.e. $E\left(\hat{\rho}^{2}(\mathbf{X})\right) \neq \rho^{2}(X)$. However, it is consistent because

$$
\lim _{n \longrightarrow \infty} \hat{\rho}^{2}(\mathbf{X})=\rho^{2}(X) \text { and } \lim _{n \longrightarrow \infty} V\left(\hat{\rho}^{2}(\mathbf{X})\right)=0
$$

The second condition is shown later in (11).

A possible approach to get rid of the bias is simply to use (3) to turn $\hat{\rho}^{2}$ into an unbiased estimator by defining the modified MRL $\hat{\rho}^{\prime}$ as

$$
\hat{\rho}^{\prime}(\mathbf{x}):=\sqrt{\frac{n}{n-1}\left(\hat{\rho}^{2}(\mathbf{x})-\frac{1}{n}\right)}=\sqrt{\frac{1}{n-1}\left(\frac{1}{n}\left|e^{i x_{1}}+\ldots\right|^{2}-1\right)}
$$

such that $E\left(\hat{\rho}^{\prime 2}(\mathbf{X})\right)=\rho^{2}(X)$, as desired. While this gives us an unbiased estimator for the squared MRL, this does not necessarily give us an unbiased estimator for the MRL. The reason for this is that the equation $(E(\hat{\rho}))^{2}=E\left(\hat{\rho}^{2}\right)$ for an arbitrary estimator $\hat{\rho}$ does not hold. On the contrary, the definition of the variance implies $(E(\hat{\rho}))^{2}=E\left(\hat{\rho}^{2}\right)-V(\hat{\rho})$, which shows that our modified MRL will underestimate the correct value. Note that the same is true for the standard deviation which is biased although its square, i.e. the empirical variance, is unbiased.

There is no easy way like that above for the squared MRL to calculate the expected value of the non-squared MRL. Moreover, it strongly depends on the distribution of the circular random variable $X$. Therefore, we simply take the square root, as usual, and apply a simulation for some special distributions to see how the expected values of the estimators behave for varying sample sizes. The results are shown in Fig. 1. The variance-corrected MRL shown in this figure will be introduced later.

One can see that the conventional MRL overestimates the correct value while the modified version underestimates it, as expected. Especially for small MRLs the modified estimator is 

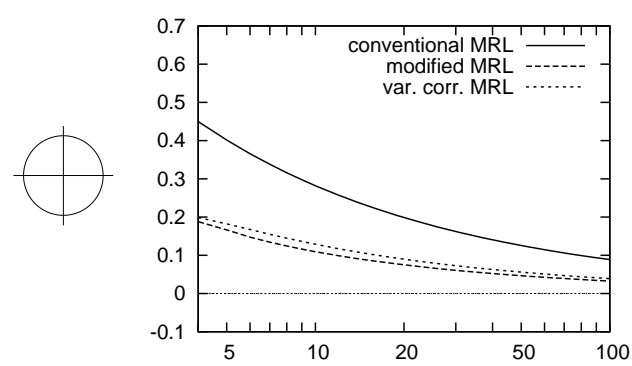

(a) uniform distribution

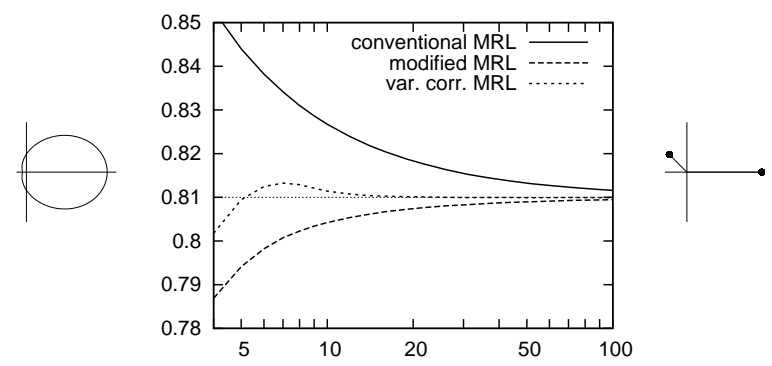

(c) von Mises distribution, $\kappa=3$

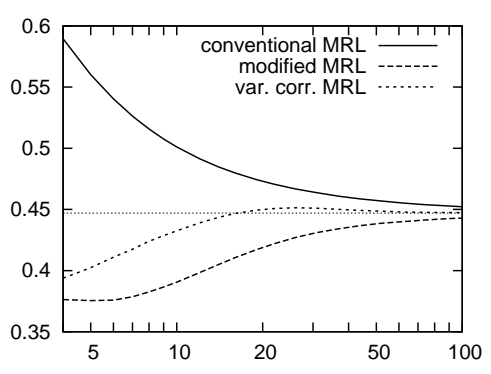

(b) von Mises distribution, $\kappa=1$

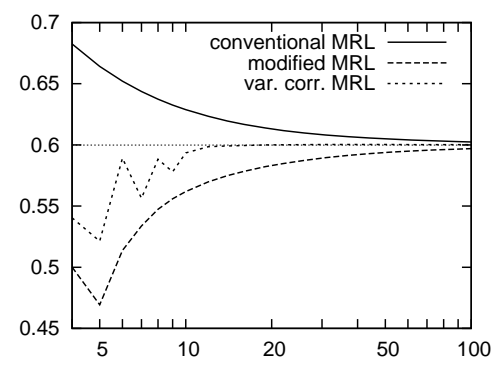

(d) discrete distribution

Figure 1. Expected values of the MRL and modified MRL estimators for increasing sample size $n$

better. But also for greater MRLs it can beat the conventional one, as Fig. 1 (c) shows. However, the conventional MRL seems to be more robust for discrete or "jumpy" distributions.

Note that the simulation can experience negative values for $\hat{\rho}^{\prime 2}(\mathbf{x})$ due to the subtraction of $\frac{1}{n}$, which leads to problems when extracting the root. In such a case, 0 is substituted, which adds a positive bias, especially for the uniform distribution.

\section{Variance and Mean Squared Error}

In linear statistics, the deviation of the average $\bar{x}$ of a sample $\mathbf{x}$ from the expected value $\mu$ is measured by its variance which can in turn be derived from the variance of the population by $V(\bar{X})=\frac{1}{n} V(X)=\frac{1}{n} \sigma^{2}$. Accordingly, the variance of the average can be estimated by means of the sample variance by $\hat{V}(\bar{x})=\frac{1}{n} s^{2}$. A similar relation can be found for the variance of the variance estimator $s^{2}$ by $V\left(s^{2}\right)=\frac{1}{n}\left(\mu_{4}-\frac{n-3}{n-1} \sigma^{4}\right)$, where $\mu_{4}$ is the fourth central moment. This suggests that the variance of all these estimators decreases with an order of $O\left(\frac{1}{n}\right)$. Our goal is now to find analogous statistics for $\hat{\rho}$ and $\hat{\rho}^{2}$.

To measure how much the MRL can deviate from its estimator, we will look at the variance and the mean squared error of the estimators. While our new modified MRL estimator $\hat{\rho}^{\prime}$ is unbiased in its squared version, variance and mean squared error will differ for the nonsquared version and for the standard estimator. 


\subsection{Variance of the Squared Standard Estimator}

Since the squared estimator is easier to handle, we will first look at the variance of the squared estimator, following [1, Ch. Moments of R]. It can be calculated by

$$
V\left(\hat{\rho}^{2}\right)=E\left(\hat{\rho}^{4}\right)-E\left(\hat{\rho}^{2}\right)^{2} .
$$

While we have already derived $E\left(\hat{\rho}^{2}\right)$ in (3), we have to do something similar for $E\left(\hat{\rho}^{4}\right)$. We obtain

$$
\begin{aligned}
E\left(\hat{\rho}^{4}(\mathbf{X})\right)= & E\left(\left(\frac{1}{n^{2}} \sum_{j=1}^{n} \sum_{k=1}^{n} e^{i X_{j}} e^{-i X_{k}}\right)^{2}\right) \\
= & E\left(\frac{1}{n^{4}} \sum_{j=1}^{n} \sum_{k=1}^{n} \sum_{l=1}^{n} \sum_{m=1}^{n} e^{i X_{j}} e^{-i X_{k}} e^{i X_{l}} e^{-i X_{m}}\right) \\
= & \frac{1}{n^{3}}\left((n-1)(n-2)(n-3) \rho^{4}(X)+4(n-1)^{2} \rho^{2}(X)+\right. \\
& 2(n-1)(n-2) \varsigma(X)+(n-1) \tau(X)+(2 n-1))
\end{aligned}
$$

after considering all possibilities for $j, k, l$ and $m$ to be equal or unequal, where

$$
\begin{aligned}
\varsigma(X) & =\Re\left(E\left(e^{i 2 X_{1}} e^{-i X_{2}} e^{-i X_{3}}\right)\right)=\Re\left(E\left(e^{i 2 X}\right) E\left(e^{-i X}\right)^{2}\right) \quad \text { and } \\
\tau(X) & =E\left(e^{i 2 X_{1}} e^{-i 2 X_{2}}\right)=\rho^{2}(2 X) .
\end{aligned}
$$

By substituting this in (6), we get

$$
\begin{aligned}
V\left(\hat{\rho}^{2}\right) & =\frac{n-1}{n^{3}}\left(2(n-2) \rho^{2}-(4 n-6) \rho^{4}+2(n-2) \varsigma+\tau+1\right) \\
& =\frac{v_{1}}{n}+\frac{v_{2}}{n^{2}}+\frac{v_{3}}{n^{3}}
\end{aligned}
$$

where

$$
v_{1}=2 \rho^{2}-4 \rho^{4}+2 \varsigma
$$

is the principal measure of inaccuracy of MRL estimation. For $n$ large enough and $v_{1}$ not near 0 , it is reasonable to approximate $V\left(\hat{\rho}^{2}\right)$ by $\frac{v_{1}}{n}$. Note that while in linear statistics $V(\bar{X})=\frac{V(X)}{n}$, in our case $v_{1}$ plays a similar role for $\rho^{2}$ as $V(X)$ does for $\bar{X}$.

$v_{1}$ depends only on the distribution and takes values between $0 \leq v_{1} \leq 1$, which is not so easy to see. To prove that $v_{1} \leq 1$, we first show that $\varsigma \leq \rho^{2}$ :

$$
\begin{aligned}
\varsigma(X)=\Re\left(E\left(e^{i 2 X}\right) E\left(e^{-i X}\right)^{2}\right) \leq\left|E\left(e^{i 2 X}\right) E\left(e^{-i X}\right)^{2}\right|=\left|E\left(e^{i 2 X}\right)\right|\left|E\left(e^{-i X}\right)\right|^{2} \\
\leq\left|E\left(e^{-i X}\right)\right|^{2}=\rho^{2}(X) .
\end{aligned}
$$


With that it follows easily that

$$
v_{1}=2 \rho^{2}-4 \rho^{4}+2 \varsigma \leq 2 \rho^{2}-4 \rho^{4}+2 \rho^{2}=4 \rho^{2}\left(1-\rho^{2}\right) \leq 4 \frac{1}{2}\left(1-\frac{1}{2}\right)=1 .
$$

To prove that $v_{1} \geq 0$, we just consider that $V\left(\hat{\rho}^{2}\right)$ would become negative for large enough $n$ if $v_{1}$ was negative, since the $v_{2^{-}}$and $v_{3}$-parts decrease faster than the $v_{1}$-part (see (11)). Of course, that is not possible, so $v_{1}$ must not be negative.

$v_{1}$ actually can take the values 0 and $1 . v_{1}$ is 0 simply if $\rho=0$, e.g. for the uniform distribution. The case $v_{1}=1$ can be constructed with a discrete distribution with two possible angles 0 and $\pi$, where $P(X=0)=\frac{1}{4}(2+\sqrt{2})$ and $P(X=\pi)=\frac{1}{4}(2-\sqrt{2})$, so that $\rho^{2}=\varsigma=\frac{1}{2}$ and thus $v_{1}=1$. This case is similar to our discrete test distribution, so we will see that the latter will have a high variance in the simulation that is to follow.

To find an estimator for the variance $V\left(\hat{\rho}^{2}\right)$, we first find estimators for $\varsigma$ and $\tau$ :

$$
\begin{aligned}
& \hat{\varsigma}(\mathbf{x})=\Re\left(\left(\frac{1}{n} \sum_{k=1}^{n} e^{i 2 x_{k}}\right)\left(\frac{1}{n} \sum_{k=1}^{n} e^{-i x_{k}}\right)^{2}\right), \\
& \hat{\tau}(\mathbf{x})=\left|\frac{1}{n} \sum_{k=1}^{n} e^{i 2 x_{k}}\right|^{2},
\end{aligned}
$$

which have the following expected values:

$$
\begin{aligned}
& E(\hat{\varsigma}(\mathbf{X}))=\frac{1}{n^{3}}\left(n(n-1)(n-2) \varsigma(X)+2 n(n-1) \rho^{2}(X)+n(n-1) \tau(X)+n\right), \\
& E(\hat{\tau}(\mathbf{X}))=\frac{n-1}{n} \tau(X)+\frac{1}{n} .
\end{aligned}
$$

Now we can find a linear combination $\hat{V}\left(\hat{\rho}^{2}\right):=a \hat{\rho}^{4}+b \hat{\rho}^{2}+c \hat{\varsigma}+d \hat{\tau}+e$ such that $E\left(\hat{V}\left(\hat{\rho}^{2}\right)\right)=$ $V\left(\hat{\rho}^{2}\right)$ (as in (6)) is fulfilled. By comparing the coefficients of $\rho^{4}, \rho^{2}, \varsigma, \tau$ and 1 , we obtain a system of five equations that gives us the solution

$$
\begin{aligned}
\hat{V}\left(\hat{\rho}^{2}\right)=\frac{2(n+1)}{n(n-3)} \hat{\rho}^{2}-\frac{4 n-6}{(n-2)(n-3)} \hat{\rho}^{4}+\frac{2(n-1)}{(n-2)(n-3)} \hat{\varsigma} & \\
& -\frac{n-1}{n(n-2)(n-3)} \hat{\tau}-\frac{1}{n(n-2)},
\end{aligned}
$$

which is an unbiased estimator for the variance of the squared MRL estimator.

However, this variance estimator is only a good indicator of the quality of MRL estimation if it does itself not deviate too much from the true variance. Therefore, we conduct a simulation to see how the estimated variances spread around their mean. Fig. 2 shows results for our four distributions. To give an impression of the distribution of the estimated variance values, the median, quartiles and lowest and highest decile is shown. Additionally, all variances are multiplied by the sample size $n$ because, as for the linear case, the variance decreases with $n$ by an order of $\frac{1}{n}$. In this way, the plot can show more details.

The fact that the lower quartile remains below zero for the uniform distribution indicates that the variance estimator does not make sense for small MRLs since there is a non-negligible probability that the variance estimator reports a very small (or even negative) variance whereas the true variance is much higher. For all other distributions, the estimator seems 


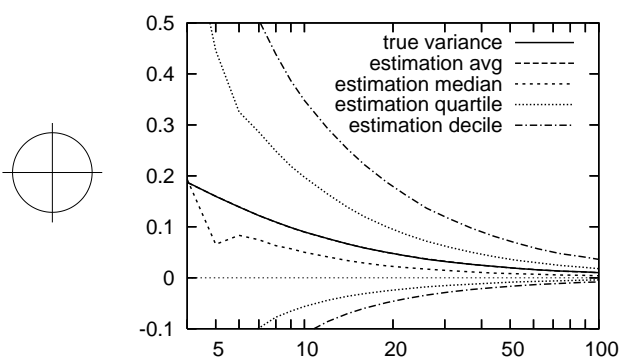

(a) uniform distribution

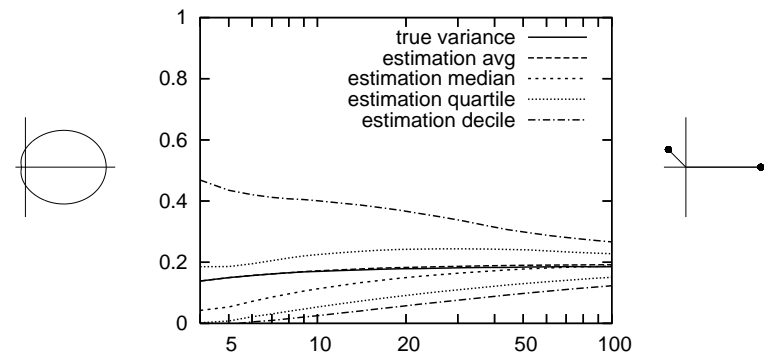

(c) von Mises distribution, $\kappa=3$

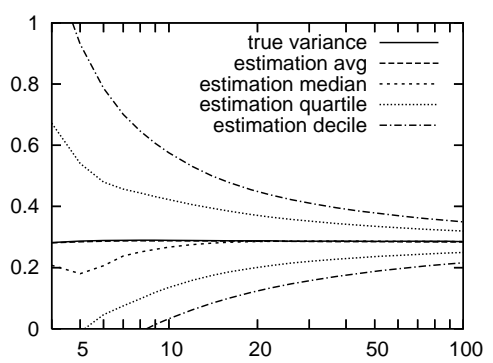

(b) von Mises distribution, $\kappa=1$

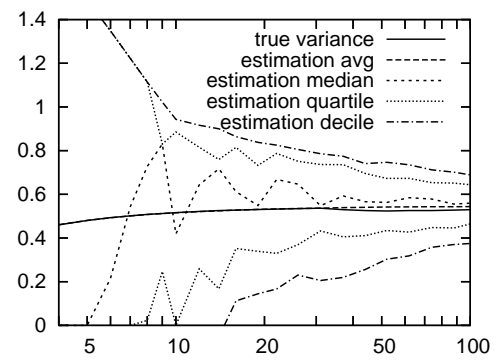

(d) discrete distribution

Figure 2. Variance estimation for the squared standard MRL estimator $\hat{\rho}^{2} \cdot n \hat{V}\left(\hat{\rho}^{2}\right)$ is shown since $\hat{V}\left(\hat{\rho}^{2}\right) \rightarrow 0$.

to be trustworthy if the sample size is at least 20, say. With increasing $n$, the estimation accuracy increases. Of course,

$$
n \hat{V}\left(\hat{\rho}^{2}\right) \stackrel{n \rightarrow \infty}{\longrightarrow} v_{1}
$$

There is also the possibility to estimate $v_{1}$ by

$$
\hat{v}_{1}:=2 \hat{\rho}^{2}-4 \hat{\rho}^{4}+2 \hat{\varsigma}
$$

and then approximate $\hat{V}\left(\hat{\rho}^{2}\right)$ by

$$
\hat{V}\left(\hat{\rho}^{2}\right) \approx \frac{\hat{v}_{1}}{n},
$$

which also gives quite good results.

\subsection{Variance of the Non-Squared Standard Estimator}

It is not possible to find, by the same means, an estimator for the variance of the non-squared estimator $\hat{\rho}$, i.e. the square root of $\hat{\rho}^{2}$. Therefore, we will try to transform $\hat{V}\left(\hat{\rho}^{2}\right)$ properly to get a good, if not unbiased, estimator $\hat{V}(\hat{\rho})$.

The first idea is to apply the so-called $\delta$-method [3, p. 77], i.e., if $\hat{\rho}^{2}$ deviates by $\epsilon$, then $\hat{\rho}$ deviates approximately by $a \epsilon$ where $a$ is the slope of the square root function at $\hat{\rho}^{2}$, i.e. $a=\frac{1}{2 \hat{\rho}}$. In this way, we obtain $\hat{V}(\hat{\rho})=\frac{\hat{V}\left(\hat{\rho}^{2}\right)}{4 \hat{\rho}^{2}}$. This estimator is consistent because the 


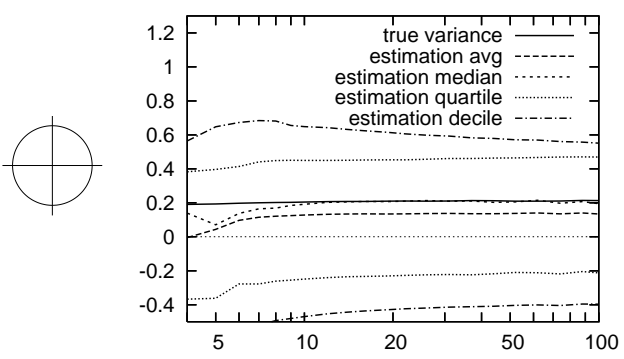

(a) uniform distribution

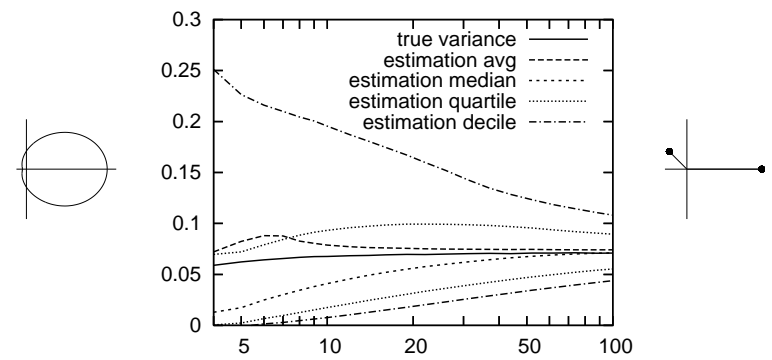

(c) von Mises distribution, $\kappa=3$

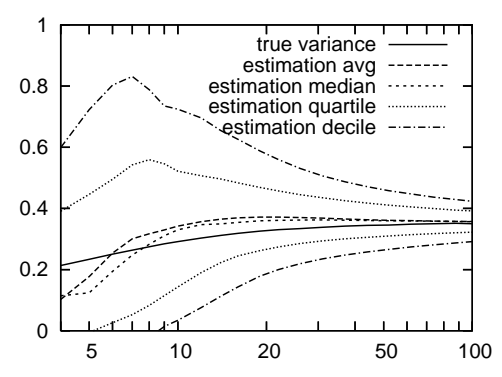

(b) von Mises distribution, $\kappa=1$

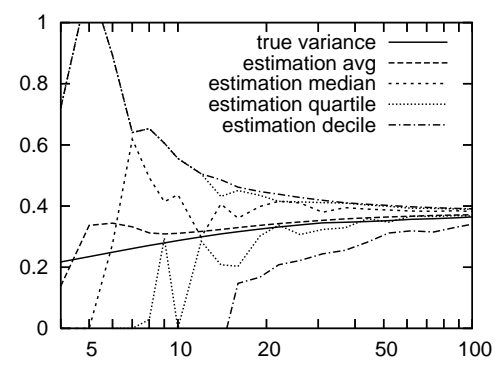

(d) discrete distribution

Figure 3. Variance estimation for the non-squared standard MRL estimator $\hat{\rho} . n \hat{V}(\hat{\rho})$ is shown since $\hat{V}(\hat{\rho}) \rightarrow 0$.

relevant region of the $\hat{\rho}^{2}$ values shrinks as $n$ grows, and the square root function can be approximated within this region by a straight line, with increasing precision. However, for smaller $n$ an accidentally small value of $\hat{\rho}^{2}$ (which can as well be negative, as we have seen) would make $a$ very large since the slope of the square root function tends to infinity near zero. This makes the proposed estimator very unstable, as experiments show.

Therefore, we suggest a slightly different approach. Since it is not sufficient to approximate the square root function by a straight line, we should actually transform the distribution of $\hat{\rho}^{2}$ and calculate the variance of the transformed distribution. However, the distribution is unknown, so we assume, for reasons of simplicity, a two-valued distribution with a mean of $\hat{\rho}^{2}$ and a variance of $\hat{V}\left(\hat{\rho}^{2}\right)$. This means, we let $\hat{\rho}^{2}$ deviate by $\sqrt{\hat{V}\left(\hat{\rho}^{2}\right)}$ in positive and negative direction, then transform these two values by the square root function. The difference between the two transformed values should then approximately be equal to $2 \sqrt{V(\hat{\rho})}$. Thus, we obtain

$$
\hat{V}(\hat{\rho}):=\left(\frac{\sqrt{\hat{\rho}^{2}+\sqrt{\hat{V}\left(\hat{\rho}^{2}\right)}}-\sqrt{\hat{\rho}^{2}-\sqrt{\hat{V}\left(\hat{\rho}^{2}\right)}}}{2}\right)^{2}=\frac{\hat{\rho}^{2}-\sqrt{\hat{\rho}^{4}-\hat{V}\left(\hat{\rho}^{2}\right)}}{2} .
$$

Note that the expression $\hat{\rho}^{4}-\hat{V}\left(\hat{\rho}^{2}\right)$ can have negative values. In this case, 0 is substituted to avoid a complex square root, which amounts to limitting the variance of $\hat{\rho}^{2}$ to $\hat{\rho}^{4}$, assuming that negative values for $\hat{\rho}^{2}$ do not make much sense anyway. This adds an additional negative bias to the estimator.

We investigate the performance of this estimator in a simulation in the same way as we did for the squared estimator. Results are shown in Fig. 3. The bias of the proposed estimator is 
acceptable, compared to its deviation. The problems with the uniform distribution of course remain the same.

\subsection{Mean Squared Error of the Squared Standard Estimator}

The mean squared error can be calculated very similar to the variance

$$
\begin{aligned}
\operatorname{MSE}\left(\hat{\rho}^{2}\right) & =E\left(\left(\hat{\rho}^{2}-\rho^{2}\right)^{2}\right) \\
& =E\left(\hat{\rho}^{4}\right)-2 E\left(\hat{\rho}^{2}\right) \rho^{2}+\rho^{4} \\
& =\frac{1}{n^{3}}\left(2(n-2)^{2} \rho^{2}-(n-2)(4 n-3) \rho^{4}+2(n-1)(n-2) \varsigma+(n-1) \tau+(2 n-1)\right) \\
& =\frac{v_{1}}{n}+\frac{w_{2}}{n^{2}}+\frac{w_{3}}{n^{3}} .
\end{aligned}
$$

The principal part $v_{1}$ is the same as for the variance.

To estimate the mean squared error seems difficult because the true MRL is not known. However, it can be estimated without bias. We can apply the same strategy as for the variance. Again, we model our estimator as a linear combination $\widehat{\operatorname{MSE}}\left(\hat{\rho}^{2}\right):=a \hat{\rho}^{4}+b \hat{\rho}^{2}+$ $c \hat{\varsigma}+d \hat{\tau}+e$ such that $E\left(\widehat{\operatorname{MSE}}\left(\hat{\rho}^{2}\right)\right)=\operatorname{MSE}\left(\hat{\rho}^{2}\right)$ is fulfilled. Similarly, we obtain

$$
\widehat{\operatorname{MSE}}\left(\hat{\rho}^{2}\right)=\frac{2}{n-3} \hat{\rho}^{2}-\frac{4 n-3}{(n-1)(n-3)} \hat{\rho}^{4}+\frac{2 n}{(n-1)(n-3)} \hat{\varsigma}-\frac{1}{(n-1)(n-3)} \hat{\tau},
$$

which is indeed an unbiased estimator for the mean squared error of the standard MRL estimator. Fig. 4 shows the simulation results. Again, the uniform distribution shows a problematic deviation, making the estimated mean squared error questionable.

\subsection{Variance of the Modified Estimator}

Since the squared modified estimator $\hat{\rho}^{\prime 2}$ is just scaled by a factor of $\frac{n}{n-1}$ and the shift of $\frac{1}{n}$ has no effect on its variance, we have

$$
\hat{V}\left(\hat{\rho}^{\prime 2}\right)=\frac{n^{2}}{(n-1)^{2}} \hat{V}\left(\hat{\rho}^{2}\right)
$$

The behaviour is, of course, similar to Fig. 2 .

To estimate the variance of the non-squared modified MRL estimator, the same procedure as in (23) can be applied:

$$
\hat{V}\left(\hat{\rho}^{\prime}\right):=\frac{\hat{\rho}^{\prime 2}-\sqrt{\hat{\rho}^{4}-\hat{V}\left(\hat{\rho}^{\prime 2}\right)}}{2} .
$$

Again, the plot will look similar to Fig. 3 . 

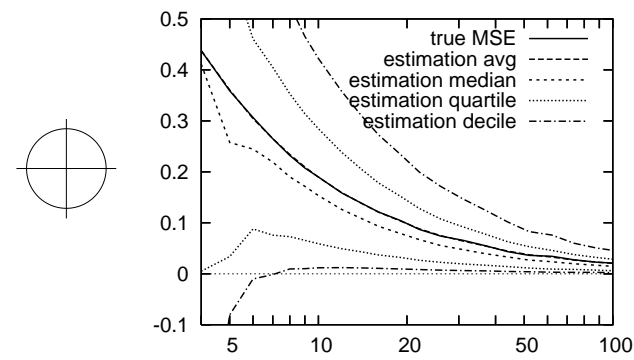

(a) uniform distribution

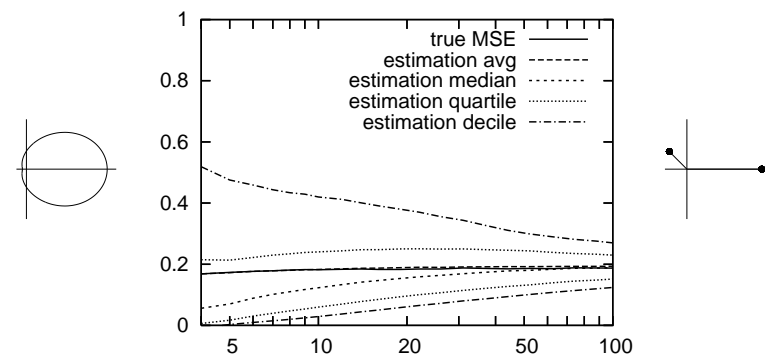

(c) von Mises distribution, $\kappa=3$

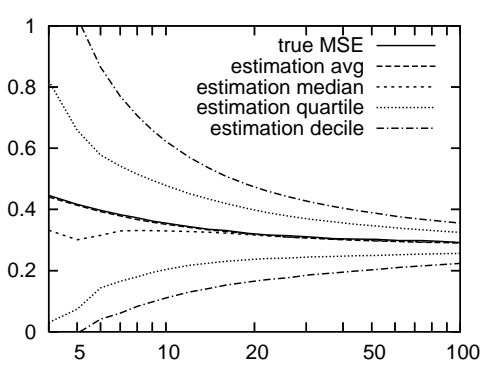

(b) von Mises distribution, $\kappa=1$

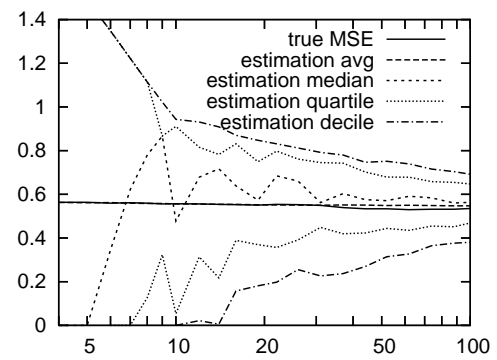

(d) discrete distribution

Figure 4. Mean squared error estimation for the squared standard MRL estimator $\hat{\rho}^{2} \cdot n \widehat{\operatorname{MSE}}\left(\hat{\rho}^{2}\right)$ is shown since $\widehat{\operatorname{MSE}}\left(\hat{\rho}^{2}\right) \rightarrow 0$.

\section{Variance-Corrected Estimator}

Now that we have a good estimate of the variance of the non-squared estimators, we can use it to construct yet another estimator with an expected value that is even closer to the correct value. By transforming the ordinary formula for the variance, we obtain

$$
V\left(\hat{\rho}^{\prime}\right)=E\left(\hat{\rho}^{\prime 2}\right)-E\left(\hat{\rho}^{\prime}\right)^{2} \Rightarrow E\left(\hat{\rho}^{\prime}\right)=\sqrt{E\left(\hat{\rho}^{\prime 2}\right)-V\left(\hat{\rho}^{\prime}\right)}=\sqrt{\rho^{2}-V\left(\hat{\rho}^{\prime}\right)} .
$$

This means that the squared expected value of the MRL estimator is reduced by the variance of the estimator. It is therefore natural to try to compensate this error by adding the estimated variance to the squared MRL estimator.

$$
\hat{\rho}^{\prime \prime}:=\sqrt{\hat{\rho}^{\prime 2}+\hat{V}\left(\hat{\rho}^{\prime}\right)}
$$

Fig. 1 shows the expected value of this variance-corrected $M R L$. One can see that it converges to the correct value much faster than the other MRL estimators.

However, the variance of the modified and variance-corrected MRL estimators is higher than that of the conventional one. Therefore, the two new estimators cannot beat the conventional one in terms of mean squared error, as is shown in Fig. 5. Only for the uniform distribution (or distributions with small MRLs) the new estimators are better. The reason for this is that negative estimations, which are implausible, have been set to 0 in the simulation, a value that produces no error if $\rho=0$. 


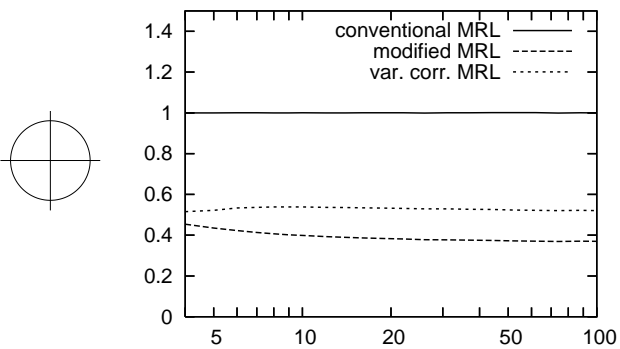

(a) uniform distribution

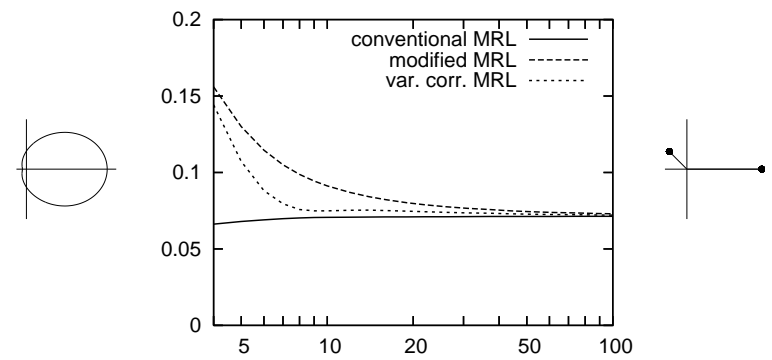

(c) von Mises distribution, $\kappa=3$

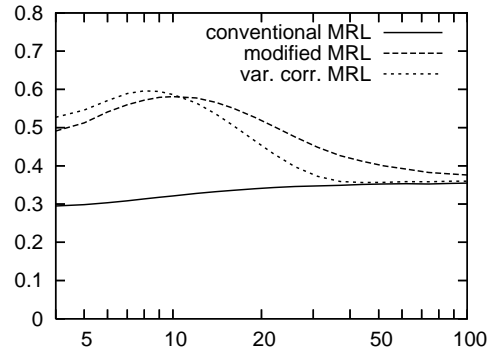

(b) von Mises distribution, $\kappa=1$

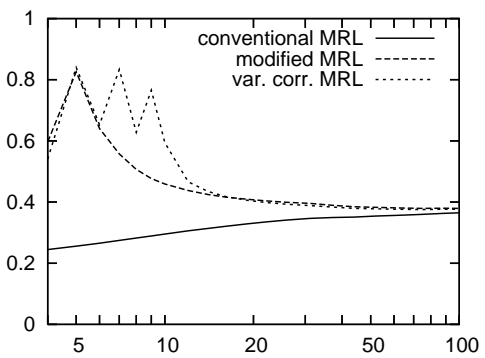

(d) discrete distribution

Figure 5. Mean squared error of the (non-squared) conventional, modified and variance-corrected MRL estimators. $n \operatorname{MSE}(\hat{\rho}), n \operatorname{MSE}\left(\hat{\rho}^{\prime}\right), n \operatorname{MSE}\left(\hat{\rho}^{\prime \prime}\right)$ is shown since $\operatorname{MSE}(\cdot) \rightarrow 0$.

\section{Estimation for a Sample Subset}

Often, samples of distinct populations have to be compared, in order to prove that the populations have different directional concentration. However, the samples may have different size, which makes the corresponding MRLs not comparable directly because bias and variance depend on the sample size. Therefore, it might be necessary to scale down the MRL of the larger sample and its variance to a subset of the size of the smaller sample, while utilizing the information of the whole larger sample.

In the linear case, the average of a subset $\mathbf{y}$ of a sample $\mathbf{x}$ is equal to the average of the total sample in terms of their expected value $(E(\bar{Y})=E(\bar{X}))$. The variance of the average of the subset is increased to $V(\bar{Y})=\frac{n}{m} V(\bar{X})$, where $n$ is the size of the total sample $\mathbf{x}$ and $m$ is the size of the subset $\mathbf{y}$. Accordingly, $\hat{V}_{m}(\bar{X}):=\frac{n}{m} \hat{V}(\bar{X})$ is a good estimator for $V(\bar{Y})$. This relation is, for instance, applied when we test if two samples belong to the same population.

Now we want to examine the corresponding procedure when estimating $\rho^{2}$. If we use the standard estimator $\hat{\rho}^{2}$, then $E\left(\hat{\rho}^{2}(\mathbf{Y})\right) \neq E\left(\hat{\rho}^{2}(\mathbf{X})\right)$ because the standard estimator is biased (see (3)). To improve $\hat{\rho}^{2}(\mathbf{y})$ we model a new estimator $\hat{\rho}_{m}^{2}(\mathbf{x})=a \hat{\rho}^{2}(\mathbf{x})+b$ such that $E\left(\hat{\rho}_{m}^{2}(\mathbf{X})\right)=E\left(\hat{\rho}^{2}(\mathbf{Y})\right)$ is fulfilled. Comparing the coefficients of $\rho$ and 1 , we obtain

$$
\hat{\rho}_{m}^{2}(\mathbf{x}):=\frac{n(m-1)}{m(n-1)} \hat{\rho}^{2}(\mathbf{x})+\frac{n-m}{m(n-1)} .
$$

However, the difference between $\hat{\rho}_{m}^{2}(\mathbf{x})$ and $\hat{\rho}^{2}(\mathbf{x})$ is small if $n$ and $m$ are large. When using the modified estimator $\hat{\rho}^{\prime 2}$, no correction at all is necessary because it is unbiased.

Now we want to improve the estimator $\hat{V}\left(\hat{\rho}^{2}\right)(\mathbf{y})$ in the same way. If we approximate $\hat{V}\left(\hat{\rho}^{2}\right)$ 


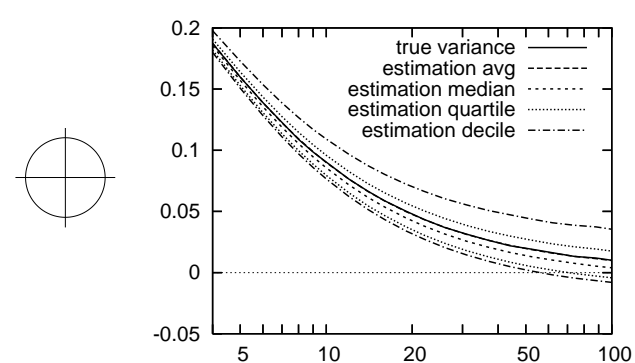

(a) uniform distribution

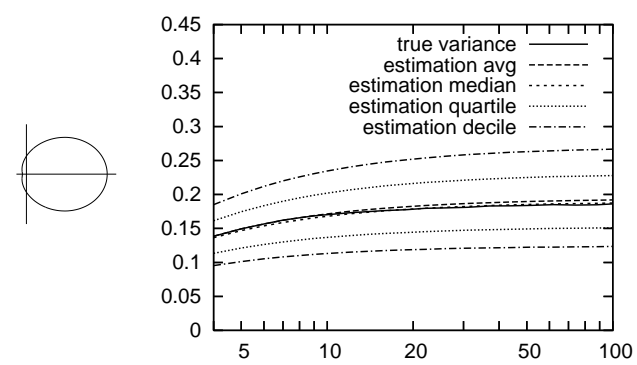

(c) von Mises distribution, $\kappa=3$

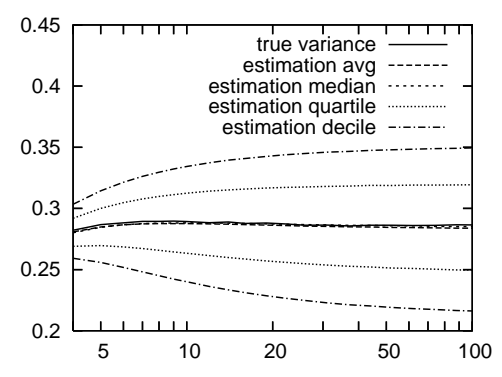

(b) von Mises distribution, $\kappa=1$
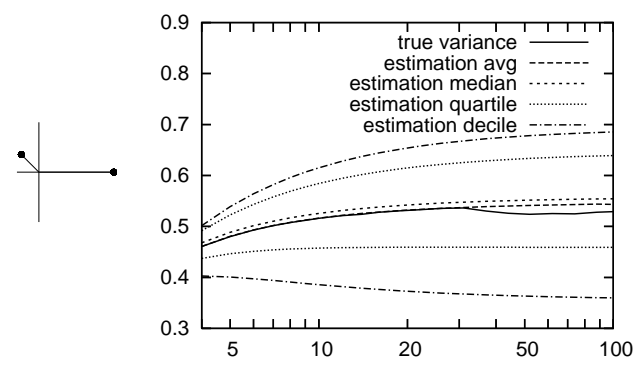

(d) discrete distribution

Figure 6. Variance estimation for a sample subset of size $m$. The total sample size is fixed $n=100$. $m \hat{V}_{m}\left(\hat{\rho}^{2}\right)$ is shown since $\hat{V}_{m}\left(\hat{\rho}^{2}\right) \rightarrow 0$.

by $\frac{\hat{v}_{1}}{n}($ see $(22))$, then it is as easy as in the linear case to state

$$
\hat{V}\left(\hat{\rho}^{2}\right)(\mathbf{y}) \approx \frac{\hat{v}_{1}(\mathbf{x})}{m}=\frac{n}{m} \hat{V}\left(\hat{\rho}^{2}\right)(\mathbf{x}) .
$$

However, to develop a more accurate estimate, we have to apply the strategy as in (29) again. We model a new estimator as a linear combination $\hat{V}_{m}\left(\hat{\rho}^{2}\right)(\mathbf{x})=a \hat{\rho}^{2}(\mathbf{x})+b \hat{\rho}^{4}(\mathbf{x})+c \hat{\varsigma}(\mathbf{x})+$ $d \hat{\tau}(\mathbf{x})+e$ such that $E\left(\hat{V}_{m}\left(\hat{\rho}^{2}\right)(\mathbf{X})\right)=V\left(\hat{\rho}^{2}(\mathbf{Y})\right)$ is fulfilled. By comparing the coefficients of $\rho^{4}, \rho^{2}, \varsigma, \tau$ and 1 , we obtain

$$
\begin{array}{r}
\hat{V}_{m}\left(\hat{\rho}^{2}\right)(\mathbf{x}):=\frac{n(m-1)}{m^{3}(n-1)(n-2)(n-3)}\left(2(m(n(n+1)-4)-2 n(n-1)) \hat{\rho}^{2}(\mathbf{x})\right. \\
-n^{2}(4 m-6) \hat{\rho}^{4}(\mathbf{x})+2 n(m n+m-2 n) \hat{\varsigma}(\mathbf{x})+(n-2 m)(n-1) \hat{\tau}(\mathbf{x}) \\
+(n-2 m)(n-3)) .
\end{array}
$$

Fig. 6 shows numerical results for a fixed total sample size of $n=100$ and varying sample subset size $m$. One can see that not only is the accuracy of the variance estimation for the total sample transfered to the estimation for the sample subset, but the accuracy even increases for decreasing sample subset size $m$. 


\section{Conclusion}

Similar to the linear variance and standard deviation, it is possible to estimate the squared circular mean resultant length (MRL) without bias, but not in general the non-squared MRL. However, near unbiased estimators can be found. As in the linear case, the unbiasedness comes at the expense of increased variance and thus, as it seems, also increased mean squared error. Therefore, estimating the MRL appears to be a trade-off between bias and estimation error. It has to be noted that the (near) unbiased estimators produce smaller values for all distributions but most so for the uniform distribution. This is important because a given big sample MRL is less likely to have emerged by chance from a uniform distribution, which makes it easier to discriminate between a unimodal and the uniform distribution.

Estimators that are an $m$-th degree polynomial of sample moments up to the $n$-th have a variance that is an $m^{\prime}$-th degree polynomial $\left(m^{\prime} \leq 2 m\right)$ in population moments up to the $2 n$-th. The squared MRL estimator is a second degree polynomial in the first complex moment of $e^{i X}$. Thus, its variance is found to be a fourth degree polynomial in the first and second complex population moments. Furthermore, an estimator for the variance of the squared MRL can be constructed through a polynomial of corresponding sample moments by choosing coefficients so that the estimator is unbiased. In a similar way, the mean squared error of the squared MRL can be calculated and estimated.

Again, the variance of the non-squared MRL cannot be estimated without bias. However, the bias is small compared to the estimation error. Generally, for the squared and the nonsquared MRL, the variance estimation is trustworthy only if the sample size and also the MRL itself is big enough. For MRLs near zero the variance estimation fails.

\section{References}

[1] K. Mardia Statistics of Directional Data, Academic Press, New York, 1972.

[2] S. Jammalamadaka and A. SenGupta Topics in Circular Statistics, World Scientific Press, Singapore, 2001.

[3] S. Jammalamadaka and U. Lund, The effect of wind direction on ozone levels - a case study, Environmental and Ecological Statistics 13 (2006), pp. 287-298.

[4] B. Schack and W. Klimesch, Frequency characteristic of evoked and oscillatory electroencephalographic activity in a human memory scanning task, Neuroscience Letters 331 (2002), pp. 107-110.

[5] N. Johnson, Paths and chains of random straight-line segments, Technometrics 8 (1966), pp. 303-317.

[6] M. Stephens, Tests for randomness of directions against two circular alternatives, J. Amer. Statist. Ass. 64 (1969), pp. 280-289. 